\title{
Two-Dimensional Optical Processing Using One-Dimensional Input Devices
}

DEMETRI PSALTIS, MEMBER, IEEE

Invited Paper

Two-dimensional optical processing architectures that are implemented with one-dimensional input spatial light modulators are reviewed. The advanced state of the art of available one-dimen sional devices and the flexibility that exists in the design of two-dimensional architectures with one-dimensional transducers leads to the implementation of the most powerful and versatile optical processors. Signal and image processing architectures of this type are discussed.

\section{INTRODUCTION}

Optical information processing systems can be configured in two or three spatial dimensions, one of the dimensions being the optical axis of the system along the general direction in which light propagates. As light propagates through the system it is modulated by input data and transformed to produce, at the output plane, a light distribution modulated by the processed data (the result of the computation). Light modulators and optical processing elements are typically placed in planes perpendicular to the optical axis. An optical processor is referir $y$ to as one- or two-dimensional (1-D or 2-D) depending 1.1 whether one or two of the transverse coordinates of the optical system are used. The majority of the early optical information processing systems were 2-D processors [1]-[3]. The 2-D space provides large parallel processing capability that gives optical processors the potential for extremely high processing power. In addition, the inherent 2-D processing capability makes optics a signal processing techrology that is uniquely suited for image processing applications, which typically require extensive computations. Indeed, the first successful application of optical information processing systems was in the area of image processing, particularly in synthetic aperture radar [2] and pattern recognition [3]. 1-D processors were developed subsequently, primarily due to the emergence of acoustooptic devices (AODs) as broadband, high-quality electronic-to-optical transducers [4]. The acoustooptic spectrum analyzer is the most widely used processor of this type, consisting simply of an AOD, a 1-D Fourier transforming lens, and a linear detector array [5]. In recent years, a new generation of 2-D optical architectures has emerged. These architectures are implemented with

Manuscript received January 5, 1984. Research on optical architectures at Caltech is supported by the Air Force Office of Scientific Research, the Army Research Office, NASA, and General Dynamics.

The author is with the Department of Electrical Engineering, California Institute of Technology, Pasadena, CA 91125, USA.
1-D input devices but are configured in three spatial dimensions. The classical 2-D processors, which are implemented with 2-D spatial light modulators (SLMs) [6], [7], process data in a relatively straightforward manner, typically using spherical lenses to perform Fourier transformations or 2-D shift-invariant operations. The implementation of 2-D processors with 1-D devices is usually less straightforward, but greater flexibility exists in designing such systems. Consequently, many new architectures have emerged in a relatively short time, in which broad-band, high-quality 1-D devices have been incorporated in the 2-D optical system to produce the most powerful and versatile optical computers to date. We will examine in this paper the methods that have been proposed for implementing such processors. Before we proceed with descriptions of specific architectures we will discuss in this introductory section general characteristics of processors of this type.

The most direct motivation for using 1-D devices is their advanced state of the art. In recent years, numerous optical devices have been developed, usually for applications other than optical information processing. Nevertheless, many of these devices have been effectively used as input or output transducers in 1-D optical processors. AODs, semiconductor light sources (laser diodes and LEDs) [8], and semiconductor detectors (photodiodes and CCDs [9], [10]) are the components that have been most widely used in this manner. The relatively small size of these devices combined with advanced computer-aided lens design techniques has led to the development of miniaturized optical systems [11]. In addition, the relatively low power consumption and cost of the devices has made it possible to implement optical processors that are advantageous not only in terms of speed but also in terms of power requirement, size, and cost. These practical considerations provide strong impetus for using these same devices to implement 2-D processors with similar properties.

The ability to configure the processor in three dimensions is a property unique to optics among signal processing technologies. It is crucial to utilize the third dimension effectively because, in general, it is this property that gives optics a clear advantage over alternate technologies. Therefore, an important question is whether 2-D optical processors that are configured with 1-D devices can have sufficient processing power, because the full potential of the three-dimensional optical system can only be realized with the classical implementation of a 2-D processor utilizing 2-D SLMs. If such a processor is configured to perform 
2-D correlations, then each of $10^{6}$ samples that are stored on a 2-D SLM at the input plane, is multiplied by $10^{6}$ samples stored on a second 2-D SLM placed in the spatial frequency plane. This massive parallelism and interconnection capability results in a processing rate equivalent to $10^{14}$ analog multiplications per second, assuming that the input SLM is updated at 100 frames per second. When the input device is 1-D, some of this parallelism is lost since only $10^{3}-10^{4}$ samples can now be represented in the input plane, at one time. The loss in parallelism, however, is compensated for by the very high bandwidth of the available 1-D devices. For instance, consider a processor that is implemented with a linear array of 100 laser diodes as the input device. Each laser diode can be separately modulated with a bandwidth equal to $1 \mathrm{GHz}$, and therefore the input data can be updated every 1 ns. The processing power in this case can exceed $10^{14}$ analog multiplications per second, if the light from each laser diode is multiplied by at least $10^{3}$ samples in the optical system. It is actually possible to multiply each laser diode with up to $10^{6}$ samples and thus, given the properties of the devices that are available, larger processing power can be obtained with a 2-D optical processor if it is implemented with 1-D devices. Notice that in the above example, the $10^{3}$ interconnections are only a small fraction of the interconnection capacity of the 2-D optical system, yet they are sufficient to yield very high processing power due to the use of the broad-band input devices.

A consequence of the reduced utilization of the interconnection capacity of the optical system is increased flexibility. The 2-D Fourier transforming lens is the primary mechanism that is used to make all the possible interconnections in a 2-D optical processor. However, only a relatively small number of linear operations can be performed with a processor based on the 2-D Fourier transform. It is in fact possible to compute a wider class of linear operations through combinations of imaging and 1-D transforming (or integrating) lenses. Furthermore, greater flexibility exists in the design of such processors, permitting the optimization of the optical architecture so that a particular operation is performed in a manner that is matched to the requirements of each application.

A second important consequence of the use of broadband transducers has been the development of "dynamic" 2-D optical processors. Time plays a passive role in a classical 2-D processor: data are placed at the input plane, they are processed by spatial integration in the optical system, and then a time interval elapses during which the last result is read out and a new block of data is entered in the optical processor. The incorporation of devices such as AODs and CCDs, which have the capability of transfering data not only into, but also through the optical system, results in dynamic optical processors in which different calculations are continuously being performed. Time integration is an example of dynamic optical processing [12], [13]. As data flow through a time integrating system, computations are continuously performed and the partial results are stored (accumulated) on the optical detector by buildup of photogenerated charge. Recently, a more deliberate utilization of the time domain has been achieved through systolic 1-D and 2-D optical processing [14]-[17], Systolic optical processing is addressed in a separate paper in this issue. A byproduct of dynamic processing is increased programma- bility in such systems. Usually, the operation performed is controlled by electrical signals externally applied and it is a relatively easy task to reprogram the processor by changing these signals with an electronic processor that is interfaced to the optical system.

In summary, practical as well as powerful 2-D optical processors can be implemented using 1-D input devices, primarily because of the advanced state of the art of the available components. Furthermore, such systems are particularly versatile due to the latitude that exists in designing the spatial configuration as well as the timing of these architectures. In the remainder of the paper we explore these issues further by discussing several processors of this type. The architectures that will be described are implemented with astigmatic optical systems, typically consisting of a combination of spherical and cylindrical lenses in conjunction with the active devices used in each case. Even within this relatively narrow framework, it is not possible to cover all the systems that have been proposed. Instead, several representative architectures were selected, partially because of their significance but also because they were judged to be suitable for conveying crucial concepts.

The processors that will be discussed are all implemented with AODs and arrays of laser diodes (LDs) or LEDs as input devices. There are other 1-D devices that can be used instead, such as the array of electrooptic modulators that was recently fabricated at Xerox [59] or the CCD spatial light modulator that is being developed at Lincoln Laboratory [60]. AODs, LEDs, and LDs are used throughout the paper because these devices have been primarily used in practice and also because they are representative of the two basic types of 1-D input devices. In a LED or LD array, each element of the array can be individually modulated, and thus an entire line of data can be entered into the optical processor, in parallel, in a time approximately equal to the inverse of the bandwidth of the individual devices ( $1 \mathrm{~ns}$ for LDs). On the other hand, data are entered serially into the optical system when an AOD is used. If the voltage applied to an AOD is temporally modulated by $f(t)$, then the light diffracted by the device is modulated by $f(t-x / v)$, where $x$ is the spatial coordinate along the direction of propagation of the acoustic wave and $v$ is the acoustic velocity. At any time, $t$, the diffracted light is spatially modulated proportionally to a portion of the electrical signal applied to the device; equivalently, at any position, $x$, the light is temporally modulated by the input signal. This dual modulation is important for the synthesis of dynamic optical processors because AODs can be used not only as input devices but also as delay lines in the optical processor.

The modulation introduced by the AOD is actually more complicated [4]; the diffracted light is Doppler shifted to the center frequency of the AOD, the finite aperture of the device places a limit on the spatial extent over which the modulation takes place, and the approximation that the modulation is proportional to the applied electrical signal is valid only if the diffraction efficiency of the device does not exceed a few percent [18]. Furthermore, the modulation properties of AODs depend strongly on the angle of incidence of the illuminating light, an aspect that becomes particularly important in the design of astigmatic systems since in these architectures AODs are often illuminated with uncollimated light [19]. All these factors must be taken into consideration in the design of a practical system. How- 
ever, since our purpose in this paper is to describe the functional operation of 2-D processor architectures, the simple form of the modulating function, $f(t-x / v)$, will be used except in cases where the additional factors play a vital part in the architecture. In architectures that are implemented with incoherent light or where operations are performed on detected signals (time integrating processors), bipolar signals are typically placed on a bias so that they can be represented by light intensity, which is a positive quantity. For clarity, the details of biasing in such systems will be suppressed.

The rest of the paper is divided in three sections. The following section examines multichannel systems. 2-D optical processors for processing 1-D signals are discussed in Section III, and the final section is devoted to optical processors capable of processing 2-D signals (images).

\section{Multichannel Processors}

The basic linear operations (Fourier transform, convolution, and correlation) can be implemented with 1-D optical processors in a variety of ways [5], [20]. The majority of these architectures are (or at least can be) implemented using AODs as electronic-to-optical transducers. Such systems are successfully used in practice even though they are in direct competition with other analog and digital 1-D signal processing technologies, primarily because of the high bandwidth of AODs, which can exceed $1 \mathrm{GHz}$. The result is a processing rate that cannot be easily achieved with alternate technologies. The processing power of a 1-D optical processor can be increased further by a factor of $10^{2}$ to $10^{3}$ by simply performing the same basic $1-D$ operation in all the available channels along the second dimension of the optical system. Such multichannel processors were proposed early, for instance by Cutrona in his classic paper in 1964 [1]. These systems are useful in applications where one wishes to process a signal in many different ways, or alternatively when the same processing operation is to be performed on a number of different signals simultaneously.

The system shown in Fig. 1 is an example of a multichannel 1-D space integrating correlator. The input signal $f(t)$ is applied to the AOD in Fig. 1. The AOD is illuminated by a coherent light source. The spherical lens $L_{1}$ is a collimating lens and the cylindrical lens $L_{2}$ focuses the light in the
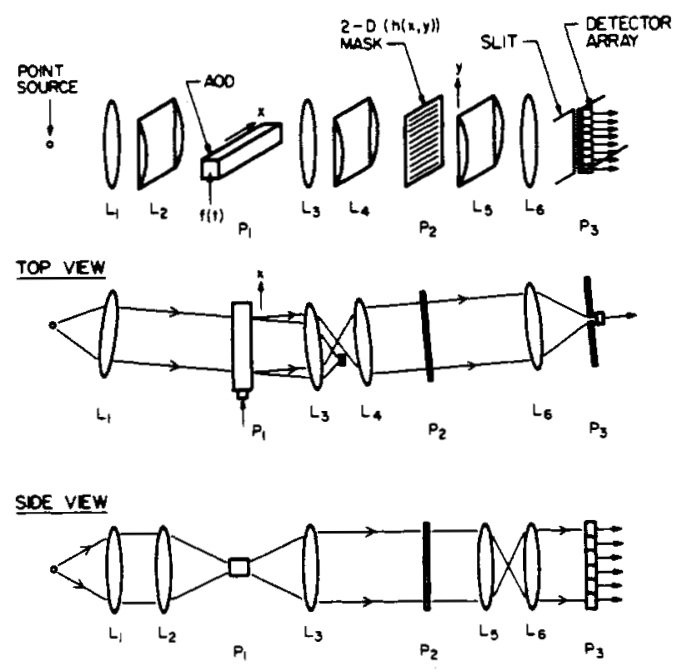

Fig. 1. Multichannel space integrating correlator. vertical direction onto the $A O D$. The amplitude of the diffracted light is modulated by $f(t-x / v)$, where $x$ is the horizontal spatial dimension and $v$ is the acoustic velocity. The undiffracted light transmitted through the AOD is blocked in the focal plane of the spherical lens $L_{3}$, whereas the diffracted light is collimated in the vertical dimension by $L_{3}$. In the horizontal dimension, $L_{3}$ and the cylindrical lens $L_{4}$ produce an image of the diffracted light at plane $P_{2}$. The light illuminating plane $P_{2}$ is therefore modulated by $f(t+x / v)$ and is uniform in the vertical $(y)$ dimension. A 2-D transparency with amplitude transmittance $h(x, y)$ is placed at $P_{2}$. The amplitude of the light transmitted through $P_{2}$ is modulated by the product $f(t-x / v) h(x, y)$. The combination of the cylindrical lens $L_{5}$ and the spherical lens $L_{6}$ image plane $P_{2}$ onto plane $P_{3}$ in the vertical dimension. In the horizontal dimension, $L_{6}$ produces the Fourier transform of the light exiting plane $P_{2}$. The amplitude of the light at the output plane $P_{3}$ is modulated by

$$
g^{\prime}\left(\omega_{x}, y, t\right)=\int f(t+x / v) h(x, y) \exp \left(j \omega_{x} x\right) d x
$$

where $\omega_{x}=2 \pi x^{\prime} / \lambda F_{6}$ is the radial spatial frequency, $\lambda$ is the wavelength of light, $F_{6}$ is the focal length of $L_{6}$, and $x^{\prime}$ is the horizontal coordinate at plane $P_{3}$. If a slit is placed at $P_{3}$, oriented vertically and centered on the optical axis, then light is transmitted through the slit only around $\omega_{x}=0$. The width of the slit is chosen to be less than $\lambda F_{6} / A$ ( $A$ being the aperture of the mask at plane $P_{2}$ ) and a detector array is placed immediately after the slit. The photocurrent that is generated at a detector element located at position $y$, is proportional to

$$
g(t, y)=\left|\int f(t+x / v) h(x, y) d x\right|^{2} .
$$

The temporal modulation of the output signal from each detector is the magnitude squared of the correlation of the input signal $f(t)$ and the signal that is recorded on the mask as a function of position $x$ at the position along the $y$ dimension where the corresponding detector element is placed. The number of parallel correlations that this system can compute is limited by the size of the parallel readout detector array that can be fabricated-in practice approximately 100 .

The multichannel, time integrating spectrum analyzer [21] shown in Fig. 2 is an example of a multichannel system that
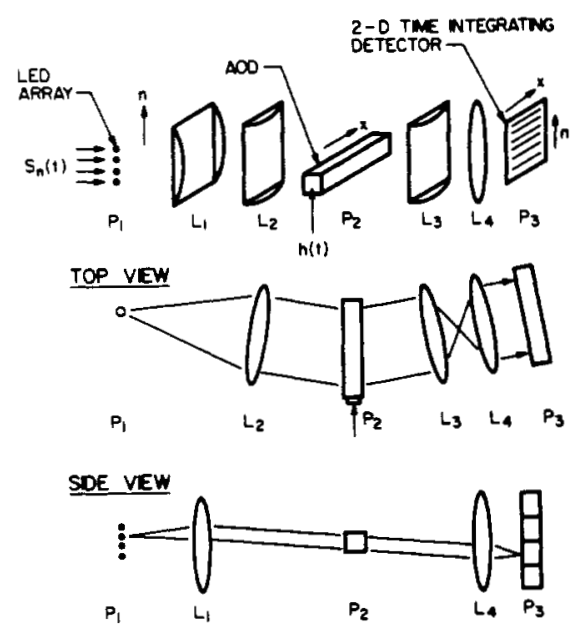

Fig. 2. Multichannel time integrating spectrum analyzer. 
can accept many signals in parallel and perform the same operation on each. A linear array of LEDs is placed in the input plane $P_{1}$ of Fig. 2. The pair of cylindrical lenses $L_{1}$ and $L_{2}$ collimate the light from each LED so that they all illuminate the AOD placed in plane $P_{2}$. The focal length of lens $L_{1}$ is chosen to be much shorter than the focal length of $L_{2}$ so that the beams illuminating the AOD are narrow in the vertical direction and wide horizontally matching the shape of the aperture of the AOD. The intensity of the $n$th LED is modulated by

$$
I_{n}=I_{0}+s_{n}(t) \cos \left(\omega_{0} t+b t^{2}\right)
$$

where $s_{n}(t)$ denotes the $n$th input signal, $\omega_{0}$ is a fixed frequency the significance of which will be discussed shortly, $b$ is a constant that has units $\mathrm{s}^{-2}$, and $I_{0}$ is a bias that is added to each signal to allow negative values to be represented by light intensity. The modulating signal applied to the AOD is $h(t)=\cos \left(\omega_{0} t+b t^{2}\right)$. When a bias is added to this signal before it is applied to the AOD, the intensity of the diffracted light is modulated proportionally to $h(t-x / v)$. Therefore, the intensity of the light diffracted by the AOD in Fig. 2 is modulated by the product of the incident intensity and $h(t-x / v)$. The cylindrical lens $L_{3}$ and the spherical lens $L_{4}$ form an image of this diffracted light in the horizontal $(x)$ direction at the output plane $P_{3}$. The lens $L_{4}$ focuses the light vertically onto the output plane or, equivalently, the combination of lenses $L_{1}$ and $L_{4}$ produce an image of the LED array in the vertical direction at the output plane. Consequently, light that originates at the $n$th LED is detected on the $n$th row of a 2-D detector array at plane $P_{3}$. The photogenerated charge on a detector element located at position $x$ at the $n$th row of the array is proportional to the time integrated intensity of the incident light:

$$
\begin{aligned}
I_{D}(x, n)= & \int I_{n}(t, x) h(t-x / v) d t+\text { bias terms } \\
= & \int s_{n}(t) \cos \left(\omega_{0} t+b t^{2}\right) \\
& \cdot \cos \left(\omega_{0} t+b(t-x / v)^{2}\right) d t+\text { bias } \\
= & (1 / 2) \int s_{n}(t) \\
& \cdot \cos \left(\omega_{0} x / v+2 b x t / v+b x^{2} / v^{2}\right) d t+\text { bias } \\
= & (1 / 2)\left|s_{n}(2 b x / v)\right| \\
& \cdot \cos \left[\omega_{0} x / v+\phi_{n}(2 b x / v)+b x^{2} / v^{2}\right]+\text { bias. }
\end{aligned}
$$

In the above equation

$$
S_{n}(\omega)=\left|S_{n}(\omega)\right| \exp \left[\phi_{n}(\omega)\right]=\int s_{n}(t) e^{-j \omega t} d t
$$

is the fourier transform of the real signal $s_{n}(t)$. Each transform is recorded as a function of position $x$ at a different row of the detector array and therefore the spectra of all input signals can be independently accessed. The magnitude of each transform modulates the amplitude of the spatial carrier in (4) whereas the phase of the transform appears as phase modulation on the same carrier. Both quantities can be obtained directly when the detector is read out, by electronically filtering the detector signal to remove the bias terms in (4) and mixing the filtered signal with $\cos \left(\omega_{0} x+b x^{2} / v^{2}\right)$ in quadrature to obtain the real and imaginary components of $S_{n}(\omega)$. This Fourier transforming algorithm, known as the "chirp- $z$ " in the digital signal processing literature [22], has proven very useful in optics since it allows the calculation of the transform of long signals with fine resolution, using temporal integration on the detector. The integration time is limited by the dark current of the detector to several milliseconds or longer if the detector is cooled. In general, the system of Fig. 2 can be used for the calculation of the spectra of many relatively narrow-band signals with fine resolution. The number of parallel channels in this case is limited by the number of LEDs (or laser diodes) that is feasible to assemble and drive in parallel. LD arrays have been fabricated monolithically with 18 elements at $15-\mu \mathrm{m}$ centers [23] and it is feasible to fabricate larger arrays either monolithically or by stacking individual modules.

\section{2-D PROCESSING OF 1-D Signals}

In this section we consider optical processors designed to process 1-D signals in both transverse spatial dimensions. Generally, more complex 1-D signal processing operations can be performed by using the two dimensions directly, resulting in optical processors that are not only computationally more powerful than 1-D processors (as are multichannel systems), but also more versatile and capable of performing a wider class of signal processing operations. In some cases the distinction between multichannel and 2-D processors is subtle. For instance, the multichannel system of Fig. 1 can also be thought of as processing its input signal in both spatial dimensions to perform a single linear operation. We will see, however, through the examples in this section that more direct utilization of the 2-D space is possible.

The first architecture considered is the vector-matrix multiplier shown in Fig. 3. This system demonstrates the
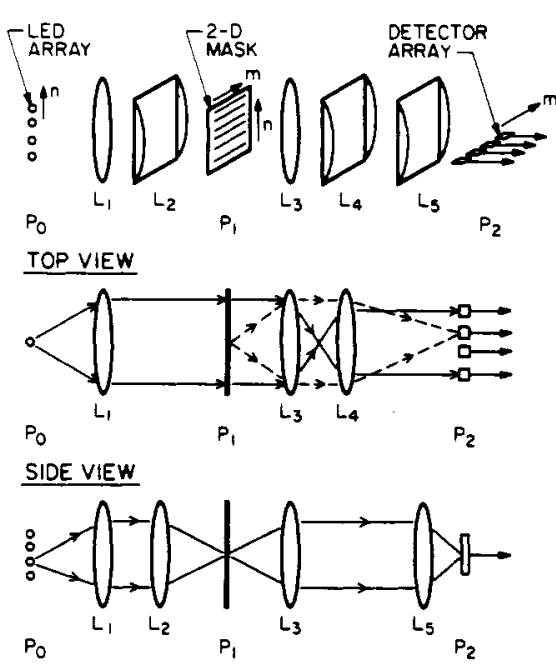

Fig. 3. Vector-matrıx multiplier, implemented with an array of LEDs.

impact that device developments have had on architectural design. The architecture in Fig. 3 was originally proposed in 1964 [1], but the full potential of this system was appreciated more than 10 years later [24]-[27], by which time broad-band source and detector arrays had become feasible. We examine here the implementation proposed by 
Goodman et al. [24]. An array of LEDs is positioned vertically at the input plane $P_{0}$ in Fig. 3 . The intensity of the light emitted by the $n$th LED is denoted by $A(n)$. The spherical lens $L_{1}$ collimates the light from each LED in the horizontal direction and the combination of $L_{1}$ and the cylindrical lens $L_{2}$ produce an image of the LED array at plane $P_{2}$ in the vertical direction. Consequently, the 2-D mask placed at $P_{2}$ is illuminated uniformly in the horizontal direction and each row of the mask is illuminated by a different LED. The intensity transmittance of the mask is denoted by $B(n, m)$, which signifies that a pixel of the mask with area $(D x \cdot D y)$, located at coordinates $x=m \cdot D x$ and $y=n \cdot D y$, has average transmittance $B(n, m)$. The mask is imaged in both dimensions onto the output plane $P_{2}$. In the horizontal dimension, the spherical lens $L_{3}$ and the cylindrical lens $L_{4}$ produce a relatively large image of the mask so that each column of the mask is imaged onto a separate element of a linear detector array placed at $P_{2}$. In the vertical dimension, $L_{3}$ and $L_{5}$ produce a demagnified image of the mask that is smaller in the vertical direction than the height of each detector element. Therefore, the photogenerated signal at the $m$ th detector element is proportional to the total intensity transmitted through the $m$ th column of the mask:

$$
C(m)=\sum_{n}^{N} B(n, m) A(n)
$$

where $N$ is the number of LEDs. The operation performed in this case is the multiplication of the vector $A$, applied to the LED array, and the matrix $B$, stored on the mask. Equivalently, it is a 1-D linear operation with a space-variant kernel. The use of the second dimension of the optical system provides in this architecture the capability to perform a wider class of operations, since it is only possible to implement shift-invariant operations and the Fourier transform with 1-D systems. It is interesting to calculate the processing power of this relatively simple optical processor. The size of the vector and the matrix that can be processed is limited by the number of elements in the LED and detector arrays. For arrays with 100 elements, $10^{4}$ analog multiplications are performed by the optical system each time a new vector is entered and the product read out by the detector. If the LEDs and the detectors have a 10-ns response time, the resulting processing rate is $10^{12}$ analog multiplications per second. The fact that this large processing power can be obtained with such a simple optical system, with which computations can be performed with relatively high accuracy, has raised the possibility of performing numerical operations optically.

Vector-matrix products can also be formed with the architecture shown in Fig. 4 that has been proposed by

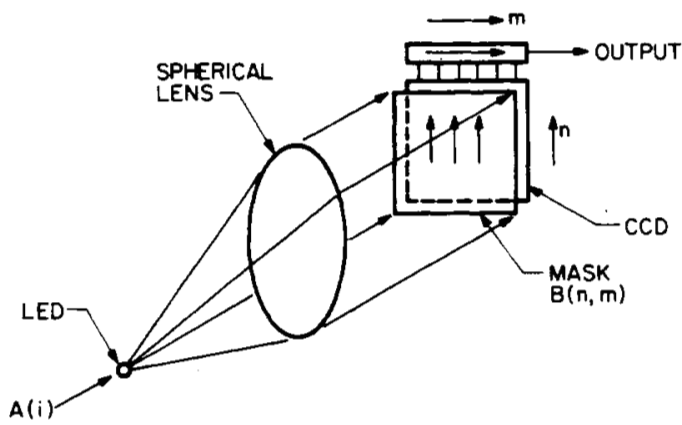

Fig. 4. Vector-matrix multiplier, implemented with a single LED and a 2-D CCD detector.
Monahan et al. [28]. In this case a single LED is used that is collimated by a spherical lens to uniformly illuminate a 2-D transparency with intensity transmittance $B(n, m)$, where $n$ and $m$ are integers denoting the position of each pixel of the mask in the two spatial dimensions. The intensity of the LED is successively modulated by the elements of the vector $A(i)$, where $i$ is an integer. The intensity of the light transmitted through the mask is modulated by the product $A(i) B(n, m)$. The light is detected by a 2-D CCD array placed immediately after the mask. There is a one-to-one correspondence between the pixels of the mask and the detector elements of the CCD and therefore the charge that is photogenerated at the $(n, m)$ detector element when the $i$ th element of the input vector is applied to the LED is $C(n, m, i)=A(i) B(n, m)$. The charge pattern stored in the $C C D$ is transferred vertically by one pixel before the next element of the vector is applied to the LED. The charge that is photogenerated during the time the LED is modulated by the $i$ th element of the input vector is transferred on the CCD by $N-i$ pixels, in the vertical direction, after all $N$ elements of the input vector have been entered in the processor. The total charge that is accumulated on the CCD after the $N$ elements of the vector are applied to the LED, is

$$
C(n, m)=\sum_{i}^{N} B(n-N+i, m) A(i) .
$$

The CCD has $n$ rows in the vertical direction and the top (Nth) row is a CCD register that transfers its contents horizontally to the output port of the device. The charge accumulated at the top row is therefore the output signal of this processor and it is found by substituting $n=N$ in (6)

$$
C(N, m)=\sum_{i}^{N} B(i, m) A(i) .
$$

Thus this system also produces at its output the product of the vector that modulates sequentially the intensity of the LED and the matrix stored in the mask. This example demonstrates how the same basic operation can be performed in different ways in a 2-D processor, each having distinct operational characteristics. The single LED and the 2-D detector array used in this architecture allow serial loading of data in the optical system, a convenient property in many applications. The parallel addressing of the system in Fig. 3, on the other hand, is useful in applications where the data naturally appear in parallel format, such as in processing signals from antenna arrays. Furthermore, the parallel input/output format of the system in Fig. 3 permits the implementation of iterative algorithms by feeding back the output signal from each detector element to the corresponding LED at the input plane of the processor [29].

Both architectures discussed thus far in this section require a 2-D transparency in addition to the input devices that are used to enter broad-band signals into the optical processor. This 2-D transparency is typically photographic film on which the matrix is permanently stored. Consequently, the matrix cannot be dynamically changed. A 2-D transparency is not, however, the only method that provides access to the 2-D space of the optical system. 2-D optical processors with dynamic spatial impulse response can be implemented using two 1-D modulators oriented perpendicular to one another. The first architecture of this type was reported by Said and Cooper [30]. The processor shown schematically in Fig. 5 is a modified version of the system that was originally proposed, in that two separate 


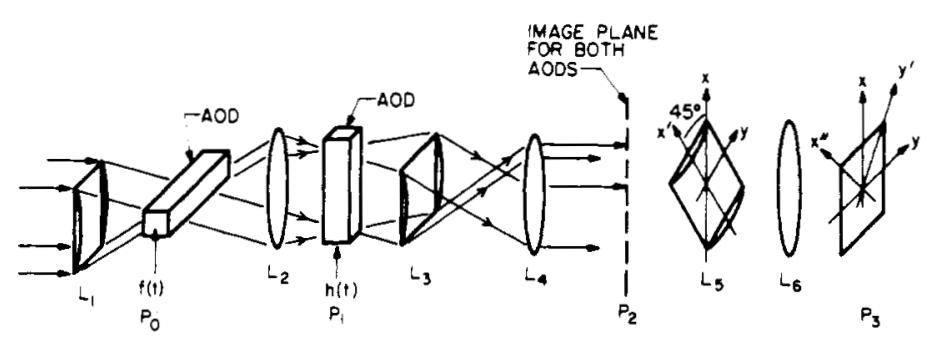

Fig. 5. Space integrating ambigurty function processor.

AODs are used here in place of a single device with two acoustic waves propagating in orthogonal directions. The monochromatic plane wave illuminating this system is focused in the vertical dimension by the cylindrical lens $L_{1}$ onto an AOD placed in the horizontal $(x)$ direction. A signal $f(t)$ is applied to the AOD and the light diffracted by the device is recollimated vertically and focused horizontally by the spherical lens $L_{2}$ onto plane $P_{1}$ where a second $A O D$ is placed oriented in the vertical $(y)$ dimension. This AOD is driven by the signal $h(t)$. In the horizontal dimension, the doubly diffracted light is collimated by the spherical lens $L_{4}$ and the combination of lenses $L_{2}$ and $L_{4}$ form an image of the light diffracted from the first $A O D$ at plane $P_{2}$. In the vertical direction, the cylindrical lenses $L_{3}$ and $L_{4}$ form the image of the light diffracted by the second AOD at $P_{2}$. The amplitude of the 2-D light distribution at $P_{2}$ is therefore modulated by $f(t-x / v) h(t-y / v)$. The astigmatic pair of lenses $L_{5}$ and $L_{6}$ image $P_{2}$ along one dimension and form the Fourier transform in the orthogonal dimension. The direction in which the cylindrical lens $L_{5}$ has focusing power is at $45^{\circ}$ with respect to the $x$ and $y$ axes and therefore the Fourier transformation is performed along a direction that is also at $45^{\circ}$ with respect to the $(x, y)$ axes. It is convenient at this point to define a new set of coordinates, $y^{\prime}$, parallel to the axis of the cylinder $L_{5}$ and $x^{\prime}$ perpendicular to $y^{\prime}$. The old coordinates are related to the new by

$$
\begin{aligned}
& x^{\prime}=(x+y) / \sqrt{2} \\
& y^{\prime}=(x-y) / \sqrt{2} .
\end{aligned}
$$

We can now readily write an expression for the amplitude of the light distribution at the output plane $P_{3}$ as the 1-D Fourier transform of the light distribution at plane $P_{2}$

$$
\begin{aligned}
g\left(u, y^{\prime}, t\right)= & \int f\left[t-\left(x^{\prime}+y^{\prime}\right) / \sqrt{2} v\right] h\left[t-\left(x^{\prime}-y^{\prime}\right) / \sqrt{2} v\right] \\
& \cdot \exp \left(2 \pi x^{\prime} u\right) d x^{\prime}
\end{aligned}
$$

where $u=x^{\prime \prime} \lambda F_{6}, x^{\prime \prime}$ is the spatial coordinate at the output plane and $F_{6}$ is the focal length of $L_{6}$. The function $g$ in the above equation has some interesting properties. If $f(t)$ $=h(t-\tau) \exp \left(j 2 \omega_{0} t\right)$ is a delayed version of the signal $h(t)$, shifted in frequency by $u_{0}$ hertz, then at $x^{\prime \prime}=$ $u_{0} \lambda F_{6} / \sqrt{2} v$ and at a time $t$ when both $f$ and $h$ are within the apertures of the AODs, $g$ as a function of position $y^{\prime}$ is the autocorrelation of $h$. The peak of this autocorrelation function occurs at $y^{\prime}=v \pi / \sqrt{2}$. This operation, known as the ambiguity function [31], is useful in radar signal processing since the range (which is proportional to $\tau$ ) and the velocity of a target (proportional to $\psi_{0}$ ) can be estimated from the location of the peak of $g$ in the $\left(u, y^{\prime}\right)$ plane. If the signals $f$ and $h$ are longer than the acoustic delay through the AODs, the ambiguity function of a different portion of the two signals is calculated at any one time. These partial ambiguity surfaces can be incoherently accumulated in time on a 2-D detector at the output plane to enhance the signal-to-noise ratio. This architecture exemplifies the distinction between multichannel and 2-D processors. The operation performed along either of the two spatial coordinates is not independently part of the final answer; only the combined processing along both transverse dimensions produces the full ambiguity surface.

The system described above is interesting in its own right, but perhaps more importantly it demonstrated for the first time a very flexible and powerful method for processing data in two dimensions: the use of two orthogonal AODs or other 1-D devices. In recent years, many configurations have been proposed using this technique [32]-[36] and it is likely that more architectures of this type will be invented. We will examine here, as an additional example, a particularly flexible architecture proposed by Kellman [37] and Turpin [38] and shown in Fig. 6. A LED is the light

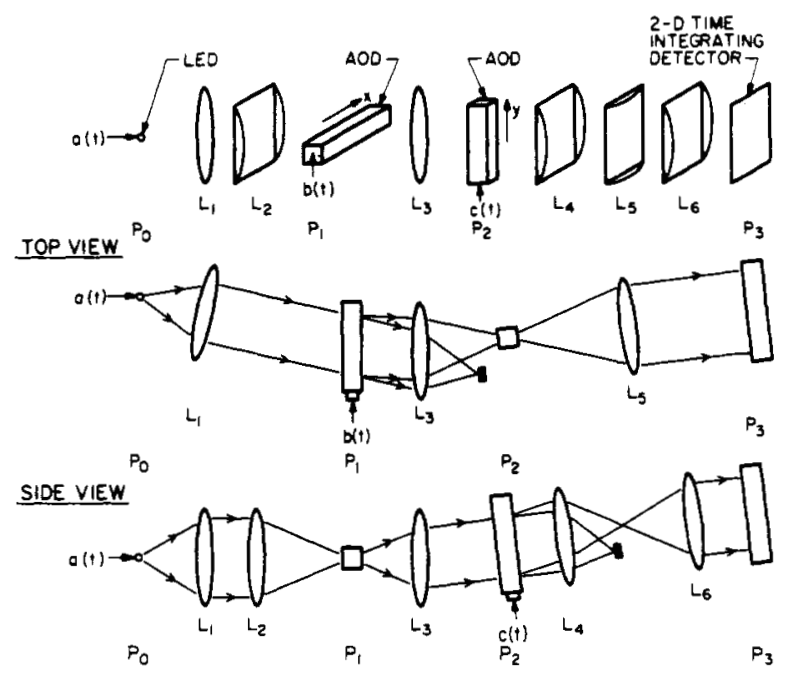

Fig. 6. Time integrating triple-product processor.

source in this system, its intensity temporally modulated by the signal $a(t)$. The first AOD, oriented horizontally in Fig. 6 , is modulated by the signal $b(t)$ and illuminated by the LED through lenses $L_{3}$ and $L_{2}$. The intensity of the light diffracted by the AOD, modulated by the product $a(t) b(t$ $-x / v)$, is expanded vertically and focused horizontally by $L_{3}$ to illuminate the second, vertically oriented AOD in Fig. 6 . The signal applied to the second AOD is denoted by $c(t)$. The doubly diffracted light is modulated by the product $a(t) b(t-x / v) c(t-y / v)$. The cylindrical lenses $L_{4}$ and $L_{6}$ produce an image of the second AOD in the vertical direction at the output plane, while $L_{3}$ and $L_{5}$ image the first AOD in the horizontal direction onto the output plane. A 2-D detector array is placed at the output plane. The photogenerated charge that accumulates at a detector ele- 
ment located at coordinates $(x, y)$ and exposed to light for $T$ seconds is proportional to

$$
d(x, y)=\int_{T} a(t) b(t-x / v) c(t-y / v) d t .
$$

This system is known as the triple product processor because the 2-D output function, $d(x, y)$, is obtained by temporally integrating the product of the three input signals. We can see from the top and side views in Fig. 6 that this processor consists of two 1-D time integrating correlators, operating simultaneously in orthogonal directions. Note the difference between this system and the multichannel time integrating processor we discussed in the previous section (Fig. 2). A correlation is performed in this case in both dimensions; the result is obtained from the combined $2-D$ processing rather than the repetition of the $1-D$ processing operation. The operation performed by this architecture always has the general form given by (9), but there is considerable flexibility since all three input signals are electronic: by altering them new operations can be performed with the same optical hardware. For instance, if $a(t)=s(t) \exp \left(j k t^{2}\right), b(t)=s(t)$, and $c(t)=\exp \left(-j k t^{2}\right)$, where $k$ is a constant, then the output, $d(x, y)$ becomes

$$
\begin{aligned}
d(x, y)= & \exp \left[-j k(y / v)^{2}\right] \int s(t) s(t-x / v) \\
& \cdot \exp [-2 j(k y / v) t] d t .
\end{aligned}
$$

The above expression is the ambiguity function of the signal $s(t)$ multiplied by a quadratic phase term (which can be removed, if necessary, by post-detection multiplication). Notice that in (10) we have allowed the input signals to be complex. In practice, complex signal processing capability is achieved by modulating in quadrature an offset carrier with the input signals and adding a bias to the modulated carrier, before the signals are applied to the AODs of the processor.

A different signal processing operation can be performed with the system of Fig. 6 , if we set $a(t)=s(t) \exp \left(j k_{1} t^{2}+\right.$ $\left.j k_{2} t^{2}\right), b(t)=\exp \left(-j k_{1} t^{2}\right)$, and $c(t)=\exp \left(-j k_{2} t^{2}\right)$ [39]. The output in this case takes the following form:

$$
\begin{aligned}
& d(x, y)=\exp \left[-j k_{1}(x / v)^{2}-j k_{2}(y / v)^{2}\right] \\
& \cdot \int s(t) \exp \left[-j\left(2 k_{1} x / v\right) t\right] \exp \left[-j\left(2 k_{2} y / v\right) t\right] d t .
\end{aligned}
$$

If we neglect the multiplicative quadratic phase term, $d(x, y)$ is the Fourier transform of the signal $s(t)$, calculated via the chirp-z algorithm, in both spatial dimensions. The spectrum is displayed in both dimensions as a function of position with $k_{1} x / 2 \pi v$ and $k_{2} y / 2 \pi v$ being the frequency variables in the $x$ and $y$ directions, respectively. The maximum frequency of $s(t)$ for which the spectrum is calculated in the $x$ and $y$ dimensions is $k_{1} A / 2 \pi v$ and $k_{2} A / 2 \pi v$, respectively, where $A$ is the aperture of both AODs in Fig. 7 (i.e., the maximum value for $x$ and $y$ ). The frequency resolution is equal to the maximum frequency divided by the number of pixels in each dimension of the 2-D detector array or the space-bandwidth product of the AODs, whichever is smaller. If $k_{1}$ is chosen so that $k_{1} A / 2 \pi v$ is equal to the bandwidth $B$ of $s(t)$, then the entire spectrum of $s(t)$ is displayed in the $x$ direction with relatively low resolution.
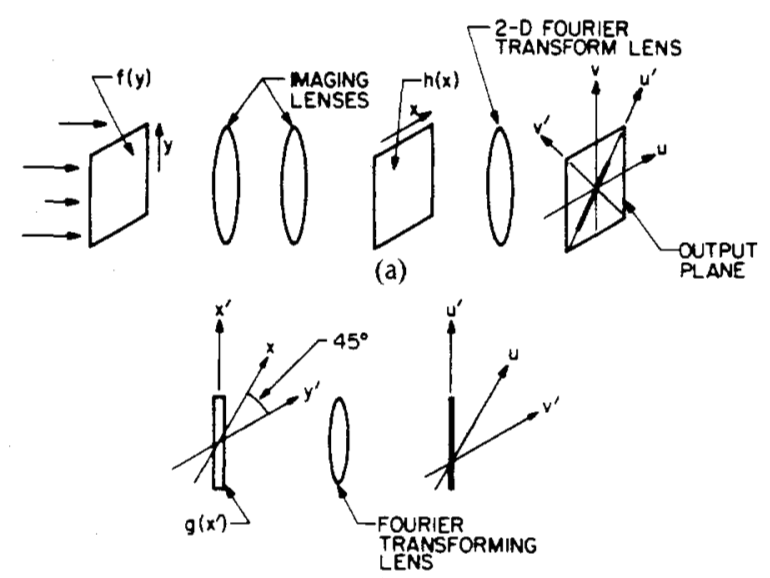

(b)

Fig. 7. Triple-correlator architecture.

If $k_{2}$ is chosen to be equal to $k_{1}$ divided by the number of pixels of the detector (let us take this number to be $10^{3}$ ), then only $1 / 10^{3}$ of the spectrum is displayed in the $y$ direction at each position $x$. The resolution in the $y$ dimension is then equal to the bandwidth of $s(t)$ divided by $10^{6}$, assuming that the integration time, $T$, on the detector is at least $10^{6} / B$ seconds. The combination of the broad-band, low-resolution transform in $x$ and the high-resolution, low-bandwidth transform in $y$ produces the 2-D "folded" spectrum or, equivalently, the 2-D raster recording of $10^{6}$ samples of the 1-D fourier transform of the signal $s(t)$. The formation of the 1-D spectrum in two spatial coordinates with this architecture, allows the display of $10^{6}$ spectral samples since 2-D detector arrays of this size are available, whereas the size of 1-D detector arrays is on the order of $10^{3}$. The triple-product processor can also be used to calculate the "folded" correlation of 1-D signals longer than the acoustic delay of the AODs used in the implementation. This is possible for signals that can be written as the product of two shorter codes [40]. The advantage of the folded correlation over a 1-D implementation is that two very long signals can be correlated and at the same time long differential delays between the two signals are permitted. Either one of these properties can be achieved with a 1-D implementation, but they can be obtained simultaneously only with the 2-D implementation.

The product of two matrices can also be computed by this same architecture using the method described by Athale et al. [41]. This is accomplished by applying the columns of the first matrix sequentially to the first AOD in Fig. 6 while the rows of the second matrix are applied to the second $A O D$. At periodic time instances, when the acoustic signals in the two AODs correspond to one complete column and row of the matrices, the LED is pulsed and the outer product between the two vectors is formed and detected at the output plane of the system. The accumulation on the 2-D detector of the outer products of all the columns of the first matrix with the corresponding rows of the second matrix, results in the formation of the product matrix. In the vector-matrix multipliers we considered earlier (Figs. 3 and 4) both spatial dimensions are used to perform an operation simpler than the product of two matrices. The reason it is possible to perform directly more complex operations with the triple-product processor is that the detector is used to store and accumulate partial results, i.e., data are processed 
in the time domain in addition to the two spatial coordinates. Interestingly, however, the triple-product processor that forms the matrix-matrix product is less powerful than the vector-matrix multiplier of Fig. 3. A matrix-matrix product can be performed with the system of Fig. 3 by repetitive vector-matrix multiplications (inner products). The number of inner products that must be computed with this approach is equal to the number of outer products that must be computed with the triple-product processor. The vector-matrix multiplication is computed by the system of Fig. 3 in a time equal to the inverse of the modulation bandwidth of the LEDs, which can be 10 ns or less. The outer product is computed in the system of Fig. 7 in the time it takes to load the AODs, typically $1 \mu \mathrm{s}$ or more. Thus the simpler system of Fig. 3 has the speed advantage. This is a consequence of the high degree of parallelism at the input and output stages of this processor, a feature however that in practice is not desirable because of the need to handle electronically many parallel broad-band channels. This tradeoff between speed and parallelism at the input and output stages of the optical system is frequently encountered in the design of optical processing systems.

We now discuss an architecture in which the 2-D space is ingeniously used to perform the correlation of three 1-D signals. The triple correlation is equivalent in the frequency domain to the product of the 1-D transforms of the three signals. All three transforms can be produced with 1-D optical systems (Fourier transforming lenses) but only double products of these transforms are produced in one dimension by detecting their interference. The triple product of the transforms can be formed if both transverse dimensions of the optical system are used, with the processor shown in Fig. 7. This system is a simplified version of an architecture proposed by Vander Lugt [42]. The first function, $f(y)$, is recorded as a 1-D transparency in the vertical direction, and it is imaged through a second horizontal transparency whose transmittance is the second function, $h(x)$. The amplitude of the light following the second transparency is modulated by the product $f(y) h(x)$. A spherical lens forms the 2-D Fourier transform of this product and produces at the output plane of the system in Fig. 7 (a) a light distribution whose amplitude is modulated by the product $F(v) H(u) . F$ and $H$ are the transforms of $f$ and $h$, respectively, and $v$ and $u$ are the spatial frequency variables at the output plane. If the $(u, v)$ coordinates are rotated by $45^{\circ}$ to obtain the new coordinates $u^{\prime}=(u+$ $v) / \sqrt{2}, v^{\prime}=(u-v) / \sqrt{2}$, the field at the output plane takes the form $F\left[\left(u^{\prime}-v^{\prime}\right) / \sqrt{2}\right] H\left[\left(u^{\prime}+v^{\prime}\right) / \sqrt{2}\right]$. At $v^{\prime}=0$ the amplitude of the field is modulated along the $u^{\prime}$ direction by the product of the two transforms. The product of the three transforms can now be formed by detecting the interference of this pattern and the transform of the third signal. The third transform is produced in a separate portion of the system, shown in Fig. 7(b). A 1-D transparency of the third signal, $g\left(x^{\prime}\right)$, is recorded in a direction that is at $45^{\circ}$ with the $(x, y)$ axes and parallel to the $u^{\prime}$ axis. The focal length of the Fourier transforming lens in Fig. $7(b)$ is $\sqrt{2}$ times longer than the one in Fig. 7(a) to compensate for the scaling that occurs from observing the spectra along the diagonal in Fig. 7(a). When the interference of the output light from the two systems in Fig. 7(a) and (b) is detected along the $u^{\prime}$ axis, one of the terms in the interference pattern consists of the product of the three transforms. The triple correlation can now be formed by inverse fourier transforming this term. In Vander Lugt's implementation all three transparencies are implemented with AODs. Therefore, there is a proportional temporal trequency shift at each spatial frequency and this makes it possible to obtain the inverse transform as the temporal signal at the output of a detector that integrates all the light along the $u^{\prime}$ axis. The particularly interesting feature of this architecture is the use of three AODs oriented in three separate directions. The use of more than two 1-D devices in nonperpendicular directions is the possibility that is only beginning to be explored and will likely result in new 2-D architectures in the future.

The systems that have been discussed here are only a portion of the possible 2-D architectures for processing 1-D signals, but through these examples it becomes apparent that the 2-D space provides not only additional processing power but also versatility. A much broader class of linear operations can be performed on 1-D signals with a 2-D processor and each operation can usually be performed in several different ways. The latitude in the design allows the optimization of the architecture to obtain the most suitable characteristics (input/output format, programmability, processing power) for each application.

\section{Processing of 2-D Signals}

It was stated in the Introduction of this paper that the 2-D processing capability of optics makes this technology particularly well suited for image processing applications. 2-D linear operations such as the Fourier transform and correlation can be directly performed optically if the input image is recorded as a 2-D transparency. When the processor is implemented with 1-D input devices, we are faced with the problem that the entire image cannot be entered simultaneously in the optical processor. Typically, images with $10^{6}$ pixels are processed, whereas the space-bandwidth product of 1-D devices is limited to approximately $10^{3}$. One possible solution to this problem is the segmentation of the image processing operation into smaller tasks, each one of which is implementable with an optical system that uses 1-D input devices. The partial results can then be stored electronically in order to synthesize the final result when all the subtasks are completed by the optical processor. Such an approach is indeed possible and feasible. In many instances, however, it is not only the processing power that makes the optical implementation attractive, but equally important, its low power requirement, size, and cost. The use of the large, high-speed electronic memory that is needed in this approach can eliminate these advantages. The need for an external memory can be avoided by using the detector of the optical system as the memory in which the partial results are temporarily stored. The partial results can be computed by spatial integration in the optical system and accumulated through temporal integration on the detector to produce the final result. A 2-D space can be constructed, in this manner, from one spatial dimension and time, in which an image can be represented and processed optically by temporal and spatial integration (TSI). Alternatively, the partial results can be computed with a time integrating optical processor, and thus a purely time integrating optical image processor can be implemented. In 
either case, the use of the 2-D optical system is essential, since the image must be processed in each of its dimensions along a separate axis of the optical system.

TSI processing was originally proposed by Bader [43] and Psaltis and Casasent [44] for spectrum analysis of 1-D signals. The spectrum of 1-D signals can be optically computed with a 1-D space or time integrating acoustooptic spectrum analyzer. The two implementations have complementary characteristics: the space integrating implementation provides wide bandwidth but relatively low resolution, whereas the time integrating system can produce a high resolution spectrum over a relatively small bandwidth. A high-bandwidth, high-resolution spectrum can be produced with a 2-D optical processor using a combination of temporal and spatial integration. The space integrating spectrum analyzer is shown in Fig. 8(a). If the signal that is

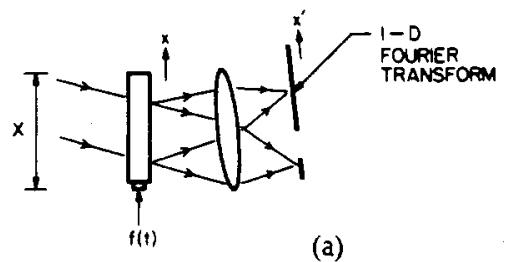

(a)

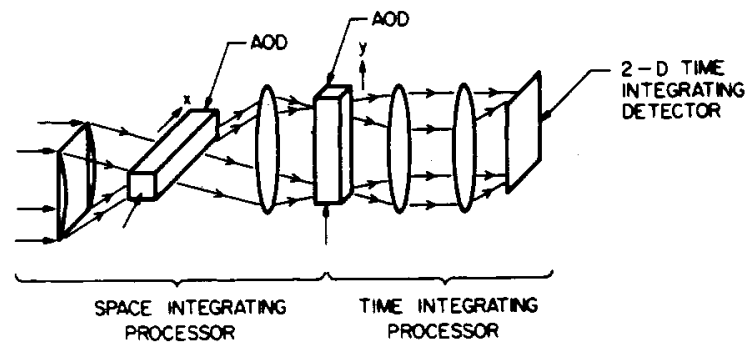

(b)

Fig. 8. Time and space integrating spectrum analyzer.

applied to the AOD is denoted by $f(t)$, then the amplitude of the light at the back focal plane of the Fourier transforming lens is modulated by

$$
\begin{aligned}
g\left(x^{\prime}, t\right) & =\int_{x} f(t-x / v) \exp (j 2 \pi u x) d x \\
& =\{f(v u) * \operatorname{sinc}(x v u)\} \exp (-j 2 \pi v u t) .
\end{aligned}
$$

$x$ in the above equation is the aperture of the AOD, $u=x^{\prime} / \lambda F$ is the spatial frequency variable, $x^{\prime}$ is the spatial coordinate at the output plane, $F$ is the focal length of the lens, and * denotes convolution. The spatial modulation at the output is the Fourier transform of $f(t), F(v u)$, smoothed by the convolution with a sinc function due to the finite aperture of the AOD. The frequency resolution is limited by the width of the main lobe of the sinc function and it is equal to $v / X$ hertz. The amplitude of the output light is also temporally modulated sinusoidally at each spatial frequency location at a temporal frequency proportional to the spatial frequency $u$. If we consider all the light incident on a single spatial frequency resolution cell (with spatial width $\lambda F / X$ ), then the bandwidth of the temporal modulation of this light is only $V / X$, equal to the frequency resolution of the space integrating processor. We can therefore use this temporally modulated light as an input to a chirp-z, time integrating processor operating in the orthogonal dimension (Fig. 8(b)) and thus produce a high-res- olution spectrum of the narrow-band temporal signal localized at each resolution element of the space integrating processor. All the coarse resolution elements can be simultaneously processed by a single multiplexed time integrating spectrum analyzer, as we discussed earlier (Fig. 2). The temporal bandwidth of the light within every one of the spatial frequency resolution cells is the same $(v / X)$, but the center frequency of the temporal modulation at each position is $n v / X$, where $n$ is an integer that increases in proportion to the position of the resolution cells of the space integrating system. The signals from all cells must be heterodyned to baseband before they can be simultaneously processed by the multichannel time integrating correlator. A pulsed light source with pulse repetition frequency $v / X$, can be used to accomplish this function. If the individual pulses are sufficiently narrow, the temporal modulation of the light source can be approximated by a series of delta functions

$$
\sum_{m} \delta(t-m X / v)=(v / X) \sum_{m} \exp [(j m v / X) t] .
$$

The amplitude of the light at the output of the space integrating system is modulated by the product of (12) and (13). We see from (13) that the periodic pulse waveform of the light source produces all the harmonics of the temporal frequency $v / X$. Therefore, at the $n$th resolution cell, the center frequency $n v / X$ of the modulation will be mixed with the $n$th harmonic of the source to produce a temporal signal at baseband. Since this happens for all $n$, there is a baseband signal at each resolution cell. The multichannel time integrating processor produces the fine frequency transform from the baseband signal in each channel while it averages to zero all nonbaseband signals and forms on the 2-D detector array at the output of the time integrating system the folded spectrum of $f(t)$.

TSI processors have been applied to other 1-D signal processing problems such as the computation of the correlation of long 1-D signals and ambiguity functions [45]-[47]. The architecture that produces the folded spectrum of a 1-D signal is of particular interest to this discussion, however, because it suggests a methodology for using TSI to do image processing. The folded spectrum was first discussed in the context of optical signal processing by Thomas [48], who showed that if a long 1-D signal is raster recorded as a 2-D transparency, then the 2-D Fourier transform of this transparency is the raster recording of the transform of the original 1-D signal (i.e., the folded spectrum). Thomas' result can be applied, in reverse, to the TSI processor: the folded spectrum produced by the TSI processor can be interpreted as the 2-D Fourier transform of the image that forms by raster recording the 1-D input signal. Each horizontal line of this image corresponds to a portion of the 1-D input signal, with duration equal to the acoustic delay through the aperture of the AOD. The 2-D Fourier transform of an image can be computed with the system of Fig. 8 , by using a TV camera to detect the image and setting the duration of each horizontal video line equal to the acoustic delay of the input $A O D$. The video from the TV camera is applied as the 1-D input signal to the processor and the 2-D Fourier transform of the image forms on the 2-D detector at the output plane in Fig. 8 . Notice that the pulse repetition frequency of the light source of the system in Fig. 8 is equal to the inverse of the acoustic delay. An alternate interpreta- 
tion of the image transforming TSI processor is as follows: each line of the input image is scanned by the TV camera and entered in the AOD; the light source is pulsed to read out the current video line which is transformed by spatial integration with the lens; the different lines of the image are sequentially scanned and entered into the optical system where the Fourier transform along the second dimension of the image is produced by temporal integration in the second dimension of the optical processor.

The TSI processing concept can be applied to a more general class of image processing operations. Specifically, it is possible to realize any 2-D linear operation that has a kernel separable in the two dimensions or shift invariant operations. 2-D linear operations with separable, but otherwise arbitrary kernel, can be implemented with the processor shown in Fig. 9 [49]. The input image is denoted

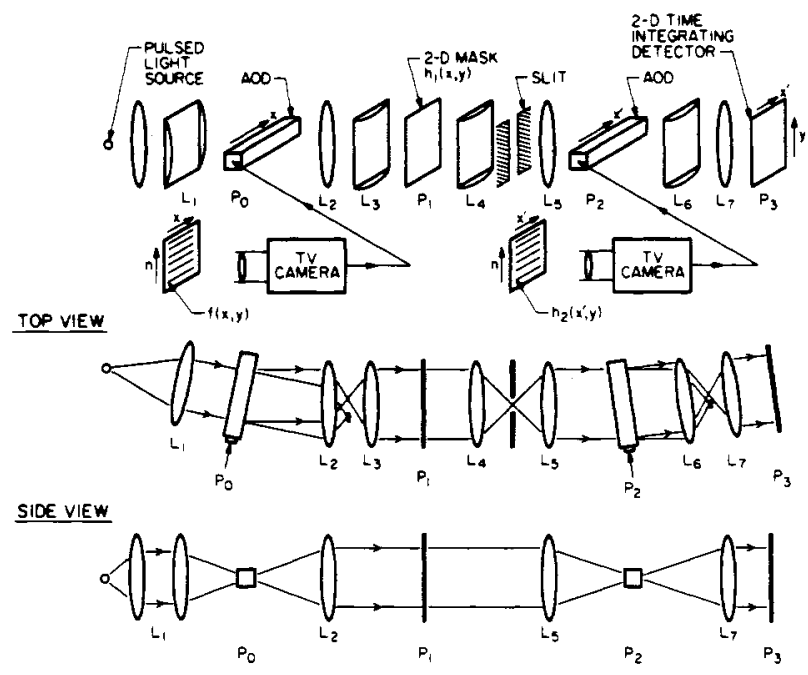

Fig. 9. Time and space integrating image processor, for the implementation of linear operations with space-variant, separable kernel.

by $f(x, n)$, where $x$ is a continuous variable along one of the dimensions and $n$ is an integer that enumerates the lines along the second dimension. A TV camera is used to scan sequentially the lines of the image $f(x, n)$, and the video is applied to the first AOD in Fig. 9. The light source is pulsed periodically to read out separately each line from the AOD. The light diffracted by the AOD is imaged in the horizontal $(x)$ direction and expanded uniformly in the vertical direction to illuminate a mask placed in plane $P_{2}$ with transmittance $h_{1}(x, y)$. The light transmitted through this transparency is imaged in the horizontal direction, by the lenses $L_{4}$ and $L_{5}$, onto a second AOD that is oriented horizontally as well. A slit is placed at the focal plane of lens $L_{4}$ that blocks high spatial frequency components in the $x$ direction. If the slit is sufficiently narrow (approximately equal to the width of one resolution spot), then the image at $P_{2}$ is spatially integrated along the $x$ direction. The second $A O D$ is modulated by the video signal from a second TV camera, which is pointed at an image $h_{2}\left(n, x^{\prime}\right)$. $x^{\prime}$ is the horizontal coordinate at the plane of the second $A O D$, and $n$ indicates the line number of the second image. The two cameras operate in synchronism, so that the acoustic signal in the second AOD is the $n$th line of the image $h_{2}$ at the instant the signal in the first $A O D$ is the $n$th line of the input image $f$. The light diffracted by the second AOD is imaged horizontally by lenses $L_{6}$ and $L_{7}$ to the output plane, where a 2-D detector array is placed. In the vertical direction, the light transmitted through the 2-D transparency, $h_{1}(x, y)$, is imaged onto the output plane by lenses $L_{5}$ and $L_{7}$. The charge that is photogenerated on the detector due to a light pulse that occurs when the $n$th line of the input image is entered in the optical processor, is proportional to

$$
g\left(y, x^{\prime}, n\right)=\left\{\int f(x, n) h_{1}(x, y) d x\right\} h_{2}\left(n, x^{\prime}\right) .
$$

The total charge that is generated and accumulated on the detector for all lines is

$$
g\left(y, x^{\prime}\right)=\sum_{n} \int f(x, n) h_{1}(x, y) h_{2}\left(n, x^{\prime}\right) d x .
$$

The operation performed by this processor, as described by (15), is a 2-D linear operation on the image $f(x, n)$ whose kernel is equal to the product of the functions $h_{1}(x, y)$ and $h_{2}\left(n, x^{\prime}\right)$. The image is processed by continuous spatial integration along one of its dimensions and by the summation of the individual lines through temporal integration on the detector, in the other dimension. Even though any separable-kernel linear operation can be implemented with the system of Fig. 9, an improved TSI architecture can often be designed for a particular separable kernel. For example, the TSI 2-D Fourier transforming system that was discussed earlier in this section, is simpler and therefore preferable to the system of Fig. 9, which can perform the same operation if $h_{1}$ and $h_{2}$ are chosen appropriately. TSI architectures for synthetic-aperture radar image formation [50], [51] and for the calculation of the moments of an image [52] have been designed by Psaltis and Wagner.

2-D shift-invariant operations are a very important class of operations that can also be implemented with TSI architectures, since such operations are useful in many image processing applications. Let $f(x, n)$ denote an input image, where $x$ is a continuous variable and $n$ is an integer denoting the line number of the image, and let $h(x, n)$ be the reference image. The 2-D correlation between $f$ and $h$ is

$$
g\left(x^{\prime}, m\right)=\sum_{n} \int f(x, n) h\left(x+x^{\prime}, n+m\right) d x .
$$

The integer $m$ in the above equation enumerates the lines of the 2-D correlation, whereas the continuous variable $x^{\prime}$ is used in the other direction. The 2-D correlation is formed by continuous integration in $x$ and summation over $n$, since the images are represented by discrete lines in our notation. The summation over $n$ is realized by temporal integration on the detector in the implementation of (16) with a TSI architecture. The 1-D integral in the $x$ direction is the correlation of each line of the input image with all lines of the reference image, i.e., for each value of $n$ the correlation is performed for all possible values of $m$. This operation is performed in a TSI processor, with a 1-D multichannel space integrating correlator in which the different lines of the reference image are stacked in the dimension orthogonal to $x$. Therefore, the index $m$ in (16) corresponds to the vertical spatial location at which a line of the correlation forms in the optical system. Notice that in (16) the 1-D correlations of the $n$th input line must be shifted in the $m$ 
direction by $n$ pixels before the summation over $n$ is performed. This shift can be implemented in the optical system with a vertical deflector that positions the 1-D correlations appropriately on the detector or, alternatively, by electronic scanning with a CCD detector array. The CCD implementation will be discussed in more detail in the following paragraph. One possible implementation of a space integrating multichannel correlator is through multiplication in the spatial frequency domain of the transform of each input line with the transforms of all the lines of the reference, which can be stored in the optical system as a 1-D Fourier transform hologram [53]. This implementation combined with a scanning CCD has been recently demonstrated experimentally at Caltech [54].

Images can also be processed with a 2-D time integrating processor, using 1-D input devices. This becomes apparent by considering the triple-product processor (an architecture in which temporal integration is performed in both dimensions) which, according to our discussion in the previous section, can be configured to calculate the folded spectrum of a 1-D signal. Therefore, the analogy between the folded spectrum of a 1-D signal and the 2-D Fourier transform can be applied in this case as well, to conclude that the tripleproduct processor can also be used for the calculation of the 2-D spectrum of an image [61]. Time integrating processors can also be configured to perform a wider class of image processing operations. We will discuss here a time integrating image correlator, shown in Fig. 10, that was

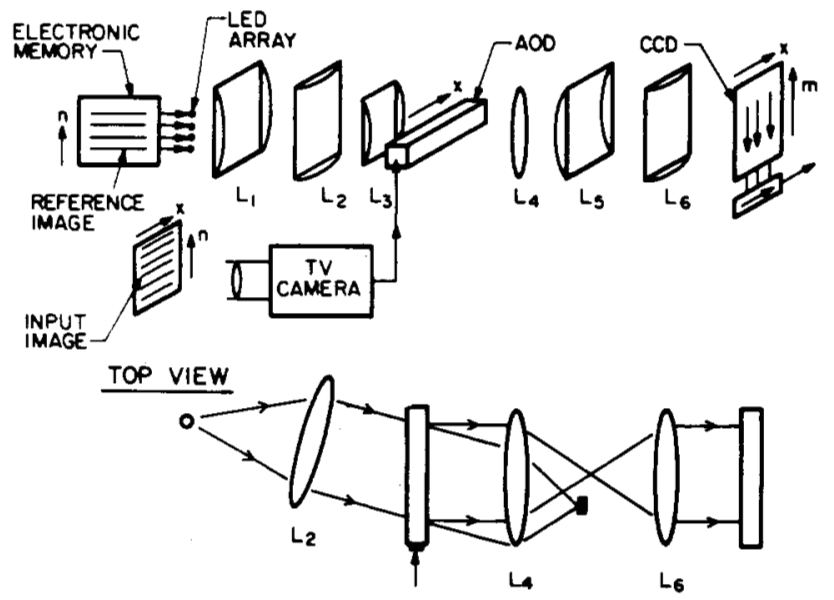

SIDE VIEW

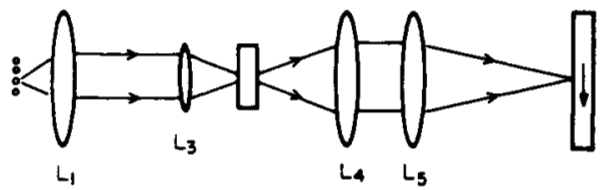

Fig. 10. Time integrating image correlator

recently described by Psaltis [55]. They system consists of a multichannel time integrating correlator in conjunction with a scanning 2-D CCD detector. A TV camera is used to raster scan the input image and the video signal is applied to the $A O D$. The reference image is stored in an electronic memory (digital or CCD). The memory is interfaced to an array of LEDs in a way that allows each line of the reference image to modulate the intensity of a separate LED. The LEDs are modulated cyclically: during the time one of the lines of the input image is traveling through the $A O D$, the intensity of the LEDs is temporally modulated by the lines of the reference image and the LED modulation is repeated every time a new input line enters the AOD. The LEDs illuminate the $A O D$ uniformly in the horizontal $(x)$ direction and the light diffracted by the $A O D$ is imaged horizontally at the output plane of the processor. In the vertical direction, the light distribution at the output plane is an image of the LED array. The charge that accumulates on the 2-D CCD detector array that is placed at the output plane in Fig. 10 during the time that the $n$th input line travels through the AOD is proportional to

$$
g(x, m, n)=\int f(t-x / v, n) h(t, m) d t .
$$

The functions $f$ and $h$ represent the input and reference images, respectively, and the integer $m$ enumerates the lines of the reference image. Since each line of the reference modulates a separate LED and each LED is located at a different vertical position, the integer $m$ in (17) also indicates the vertical position on the CCD detector. The origin of the axis of the time variable, $t$, in (17) is reset to zero at the beginning of each horizontal scan of the TV camera for notational convenience. At the completion of each horizontal scan, the 2-D charge pattern stored on the CCD is transferred vertically by one pixel. At the initiation of each horizontal scan, the electronic memory also begins to read out the reference image stored in it. When all $N$ lines of the input image have been processed, the signal that was photogenerated by the $n$th input line, has been transferred vertically on the detector by $(\mathrm{N}-n)$ pixels. The total signal that is accumulated on an element of the CCD detector array, located at horizontal position $x$ at the $m$ th row of the array, is given by

$$
\begin{aligned}
g(x, m) & =\sum_{n} g^{\prime}(x, m+n-N, n) \\
& =\sum_{n} \int f(t+x / v, n) h(t, m+n-N) d t .
\end{aligned}
$$

$g(x, m)$ is the correlation between the input image $f$ and the reference image $h$ shifted in the vertical direction by $N$ pixels. The correlation is read out by the CCD in the form of an electronic video signal that can be displayed on a TV monitor or processed further electronically. In this architecture, 1-D devices are used to enter both the input and reference images in the optical processor. Since the reference image is stored electronically, rather than optically on a 2-D SLM, it can be generated with a digital computer and stored in the memory directly. This feature can be very useful in pattern recognition applications, where typically an optimum reference is generated digitally [56] and the need to fabricate a computer-generated hologram is thereby avoided. Furthermore, this image processing architecture has unique flexibility, since the reference image can be periodically reprogrammed by the digital computer.

\section{CONCLUSION}

The design of optical processing architectures has, in a sense, gone through a complete cycle. After the early success of 2-D architectures, such as the synthetic-aperture radar processors, 1-D architectures emerged that became practical as 7-D input devices (primarily AODs) were developed. More recently the same 1-D devices have been used in a new generation of 2-D architectures. The primary motivation for the development of these architectures has proba- 
bly been the availability of the well-developed 1-D devices. In the process the field has been enriched greatly. A wider class of linear operations is possible with 2-D architectures that are implemented with 1-D devices and more flexibility exists in the way a particular linear operation can be performed. Dynamic processing (time integration and systolic processing) that is feasible with 1-D devices, coupled with the high computational power that is possible in a 2-D optical system, has resulted in the most powerful and flexible optical processors to date. Finally, the abundant processing power that is available in these architectures can be traded for increased accuracy, most notably through the use of the method of binary multiplication by analog convolution [57], [16]. This can lead to optical processors that have accuracy compatible with digital systems and thus increase the applicability of optical computing to more general problems involving linear numerical calculations.

Future architectural designs will certainly be influenced by the state of the art of optical devices. As real-time 2-D spatial light modulators become practical, they will be incorporated in 2-D architectures that use 1-D devices as broad-band input transducers and 2-D devices for massive, programmable storage in the optical system. The development of 2-D input devices that are compatible with $d y$ namic processing, such as multiple-transducer AODs [58], will make new architectural designs feasible. Nonlinear optical devices can also a have a major impact; if one- or two-dimensional arrays of optical bistable devices become feasible, then it will be possible to implement optical computers that perform not only linear, but more general computations. Such an optical computer would be more powerful than a digital computer, not because optical gates may be faster than electronic gates, but because the optical computer can be easily configured in the three-dimensional space and thus have massive parallelism and interconnection capability.

\section{ACKNOWLEDGMENT}

The author gratefully acknowledges the contributions of Dr. E. G. Paek, K. Wagner, M. Haney, and S. Venkatesh, who are his co-workers at Caltech in the research on acoustooptic image processing.

\section{REFERENCES}

[1] L. J. Cutrona, "Recent developments in coherent optical technology," in Optical and Electro-optical Information Processing, ]. I. Tippett et al., Eds. Cambridge, MA: MIT Press, 1965.

[2] E. N. Leith, "Quasi-holographic techniques in the microwave region," Proc. IEEE, vol. 59, p. 1305, 1971.

[3] A. Vander Lugt, "Signal detection by complex spatial filtering," IEEE Trans. Inform. Theory, vol. IT-10, no. 2, pp. 139-145, Apr. 1964.

[4] I. C. Chang, "Acousto-optic devices and applications," IEEE Trans. Sonics Ultrason., vol. SU-23, no. 1, p. 2, 1976.

[5] T. M. Turpin, "Spectrum analysis using optical processing," Proc. IEEE, vol. 69, no. 1, pp. 79-92, 1981

[6] D. Casasent, "Spatial light modulators," Proc. IEEE, vol. 65, no. 1 , p. 143,1977

[7] A. Tanguay, "Spatial light modulators for real time optical processing," in Future Directions for Optical Information Processing. Lubbock, TX: Texas Tech Univ., 1981.

[8] A. A. Bergh, J. A. Copeland, and R. W. Dixon, "Optical sources for fiber transmission systems," Proc. IEEE, vol. 68, no. 10, pp. $1240-1247,1980$.
[9] R. G. Smith, "Photodetectors for fiber transmission systems," Proc. IEEE, vol. 68, no. 10, pp. 1247-1253, 1980.

[10] 1. A. Hall, "Arrays and charge-coupled devices," in Applied Optics and Optical Engineering, R. R. Shannon and J. C. Wyant Eds. New York: Academic Press, 1980, ch. 8.

[11] T. Bader and P. Kellman, "Acousto-optic channelized receivers," Opt. Eng., Jan. 1984.

[12] R. M. Montgomery, "Acousto-optical signal processing system," U.S. Patent 3634 479, Jan. 1972.

[13] R. A. Sprague and C. L. Koliopoulos, "Time integrating acousto-optic correlator," Appl. Opt., vol. 15, p. 89, 1976.

[14] H. J. Caufield, W. T. Rhodes, M. J. Foster, and S. Horvitz, "Optical implementation of systolic array processing," Opt. Commun., vol. 40, p. 86, 1981.

[15] D. Casasent, J. Jackson, and C. Neuman, "Frequency multiplexed and pipelined iterative optical systolic array processors," Appl. Opt., vol. 22, no. 1, p. 115, 1983.

[16] P. S. Guilfoyle, "Systolic acousto-optic binary convolver," Opt. Eng., Jan. 1984.

[17] R. P. Bocker, H. J. Caufield, and K. Bromley, "Rapid unbiased, bipolar incoherent calculator cube," Appl. Opt., vol. 22, no. 6, p. 804, 1983.

[18] D. L. Hecht, "Multifrequency acousto-optic diffraction," IEEE Trans. Sonics Ultrason., vol. SU-24, p. 7, 1977.

[19] P. S. Guilfoyle, "Problems in two dimensions," in Proc. SPIE Conf., vol. 341-26, p. 199 (Arlington, VA, May 1982).

[20] W. T. Rhodes, "Acousto-optic signal processing: Convolution and correlation," Proc. IEEE, vol. 69, no. 1, pp. 65-79, 1981.

[21] D. Casasent and D. Psaltis, "Optical Fourier transform techniques for advanced Fourier spectroscopy systems," Appl. Opt., vol. 19, no. 12, p. 2034, 1980.

[22] A. V. Oppenheim and R. W. Schafer, Digital Signal Processing. Englewood Cliffs, NJ: Prentice-Hall, 1975, p. 321.

[23] W. T. Tsang, R. A. Logan, and R. P. Salathe, "A densely packed monolithic linear array of $\mathrm{GaAs}-\mathrm{Al}_{\mathrm{x}} \mathrm{Ga}_{7-x}$ As strip buried heterostructure laser," Appl. Phys. Lett., vol. 34, no. 2, p. 162 1979.

[24] J. W. Goodman, A. R. Dias, and L. M. Woody, "Fully parallel, high-speed incoherent optical method for performing discrete Fourier transforms," Opt. Lett., vol. 2, no. 1, p. 1, 1978.

[25] J. W. Goodman, P. Kellman, and E. W. Hansen, "Linear space-variant optical processing of 1-D signals," Appl. Opt., vol. 16, p. 733, 1977.

[26] W. T. Rhodes and J. M. Florence, "Frequency variant optical signal analysis," Appl. Opt., vol. 15, p. 3073, 1976.

[27] R. J. Marks II, J. F. Walkup, M. O. Hagler, and T. F. Krile," Space-variant processing of 1-D signals," Appl. Opt., vol. 16, p. $739,1977$.

[28] M. A. Monahan, R. P. Bocker, K. Bromley, and A. Louie, "Incoherent electro-optical processing with CCD's," in Dig. Int. Optical Computing Conf., Apr. 1975 (IEEE Cat. 75 C40941$5 \mathrm{C})$.

[29] D. Psaltis, D. Casasent, and M. Carlotto, "Iterative, color multiplexed electro-optical processor," Opt. Lett., vol. 4, no. 11 , p. 3481979.

[30] R. A. Said and D. C. Cooper, "Crosspath real-time optical correlator and ambiguity-function processor," Proc. Inst. Elec. Eng. (London), vol. 120, p. 423, 1973.

[31] A. W. Rihaczek, "Principles of high-resolution radars," Mark Resources Inc. Tech. Rep., Marina del Rey, CA, 1977.

[32] J. D. Cohen, "Ambiguity processor architectures using one-dimensional acousto-optical transducers," ProC. SPIE, vol. 180, p. 134,1979

[33] N. J. Berg, M. W. Cassedy, J. J. Abramoritz, and J. N. Lee, "Radar and communication band signal processing using time integration processors," Proc. SPIE, vol. 232, p. 101, 1980.

[34] A. Tarasevich, N. Zepkin, and W. T. Rhodes, "Matrix vector multiplier with time varying single dimensional spatial light modulation," in Proc. NASA Conf. on "Optical Information Processing," (Hampton, VA, 1981, NASA publ. 2207), p. 61.

[35] P. N. Tamura, J. J. Rebholz, T. Daehlin, and T. Lee, "Real time optical computation of the ambiguity function," Proc. SPIE, vol. 241 , p. $104,1980$.

[36] D. Psaltis, and D. Casasent, "General formulation for optical signal processing architectures," Opt. Eng., vol. 19, p. 193, 1980.

[37] P. Kellman, "Detector integration acousto-optic signal processing," in Dig. Int. Optical Computing Conf. (London, England, Sept. 1978), p. 91. 
[38] T. Turpin, "Time integrating optical processor," in Proc. SoC. of Phot. Instr. Eng. Conf. (San Diego, CA, Aug. 1978), vol. 154, p. 196.

[39] P. Kellman, "Time integrating optical signal processing," Ph.D. dissertation, Stanford Univ., Stanford, CA, June 1979.

[40] D. Casasent and G. Silbershatz, "Product code processing on a triple product processor," Appl. Opt., vol. 21, p. 2076, 1982

[41] R. A. Athale, W. C. Collins, and P. D. Stilwell, "High accuracy matrix multiplication with outer product optical processor," Appl. Opt., vol. 22, p. 368, 1983.

[42] A. Vander Lugt, "Adaptive optical processor," Appl. Opt., vol. 21, no. 22, p. 4005, 1982.

[43] T. Bader, "Acousto-optic spectrum analysis: A high performance hybrid technique," Appl. Opt., vol. 18, p. 1668, 1979.

[44] D. Psaltis and D. Casasent, "Time-and-space integrating spectrum analyzer," Appl. Opt., vol. 18, p. 3203, 1979.

[45] _. "Spread spectrum time-and-space integrating optical processor," Appl. Opt., vol. 19, p. 1546, 1980.

[46] T. R. Bader, "Coherent hybrid optical processing," Proc. SPIE, vol. 232 (Washington, DC), Apr. 1980.

[47] G. Silbershatz and D. Casasent, "Hybrid time and space integrating processor for spread spectrum applications," Appl. Opt., vol. 22, p. 2095, 1983.

[48] C. E. Thomas, "Optical spectrum analysis of large space bandwidth signals," Appl. Opt., vol. 5, p. 1782, 1966.

[49] D. Psaltis, "Acousto-optics processing of 2-D signals using temporal and spatial integration," AFOSR Rep., Calif. Inst. Technol., Pasadena, CA, May 1983

[50] D. Psaltis and K. Wagner, "Real-time optical SAR processor," Opt. Eng., vol. 21, p. 822, 1982.
[51] D. Psaltis, M. Haney, and K. Wagner, "Real-time synthetic aperture radar processing," in Proc. NASA Conf. on "Optical Information Processing" (Hampton, VA, 1983).

[52] K. Wagner and D. Psaltis, "Real-time computation of moments with acoustooptics," Proc. SPIE Conf., vol. 352-19 (San Diego, CA, 1982).

[53] D. Psaltis, "Optical image correlation using acousto-optic and charge coupled devices," Appl. Opt., vol. 21, p. 491. 1982.

[54] D. Psaltis, E. G. Paek, and S. Venkatesh, "Acousto-optic/CCD image processor," in Proc. Int. Opt. Comp. Conf. (Cambridge, MA, Apr. 1983, IEEE Cat. 83CH1880-4).

[55] D. Psaltis, "Incoherent electro-optic image correlator," Opt Eng., Jan. 1984.

[56] H. J. Caufield and W. T. Maloney, "Improved discrimination in optical character recognition," Appl. Opt., vol. 8, p. 2354, 1969.

[57] D. Psaltis, D. Casasent, D. Neff, and M. Carlotto, "Accurate numerical computation by optical correlation," Proc. SPIE, vol. 232-57, p. 151, 1980.

[58] A. Bardos, "Wideband holographic recorder," Appl. Opt., vol. 13, p. 832,1974

[59] R. V. Johnson et al., "Characteristics of the linear array total internal reflection (TIR) electro-optic spatial light modulator for optical information processing," Opt. Eng., vol: 22, p. 665, 1983.

[60] R. H. Kingston, B. E. Burke, and K. B. Nichols, "Spatial light modulation using electroabsorption in GaAs CCD," Appl. Phys. Lett., vol. 41, p. 413, 1982.

[61] W. T. Rhodes, "Space-frequency conversion for image transmission and processing," Opt. Lett., vol. 3, p. 24, 1978. 